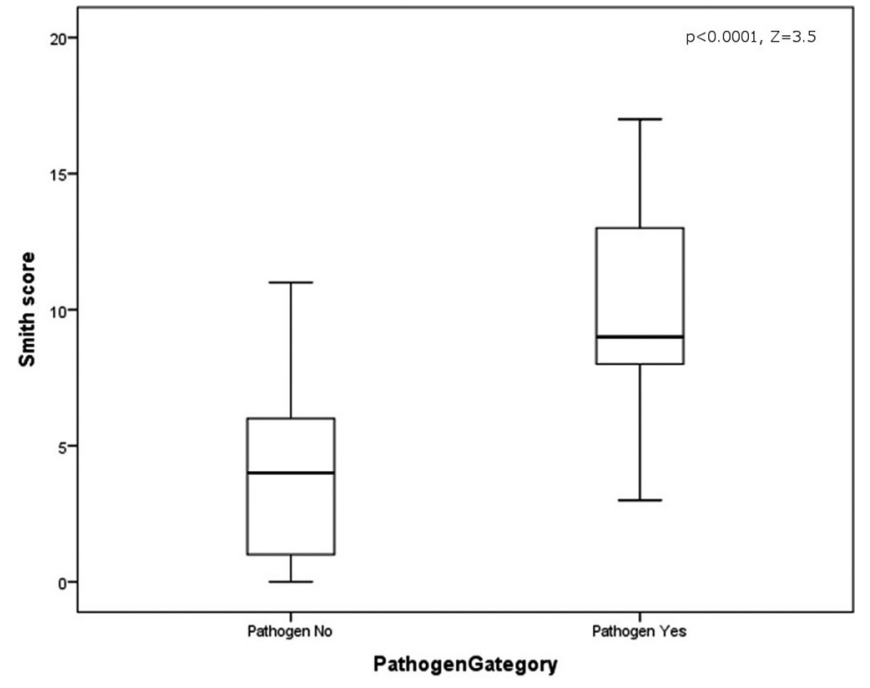

Abstract P67 Figure 1 Smith score in patients with pathogens in sputum cultures and in patients with normal flora

No correlation was found between the extent of bronchiectasis and the lung function parameters. The severity of bronchiectasis (Smith score) was correlated to the number of antibiotic cycles/year ( $p=0.002, r=0.48$ ). In addition, a lower ACT score was related with a higher asthma exacerbation rate $(\mathrm{r}=$ $0.52, \mathrm{p}=0.001$ )

Conclusion The evidence of bronchiectasis on HRCT is common in patients with severe uncontrolled asthma. Sputum production and pathogen isolation in sputum culture may indicate the presence of this comorbidity and the need of antibiotics as an additional treatment.

\section{P68 PHENOTYPING INFECTION ASSOCIATED ASTHMA: A CASE-CONTROL STUDY}

S Natarajan, R Free, A Wardlaw, S Siddiqui. Respiratory Biomedical Research Unit, Department of Infection, Immunity and Inflammation, University of Leicester, Leicester, UK

\subsection{6/thoraxjnl-2015-207770.205}

Introduction and objectives Asthmatics experiencing recurrent infective exacerbations, resembling a bacterial bronchitis, are often started on prophylactic antibiotics but this phenotype of asthma is not well understood. We sought to: a) compare asthmatics with and without recurrent infections using a case-control approach and b) phenotype asthmatics with recurrent infections to evaluate the heterogeneity within this population.

Methods We reviewed Leicester difficult asthma clinic letters within two calendar years (01/01/2013-31/12/2014) and utilised an asthma database to identify matched (age, sex, BMI and GINA treatment step) controls. Case definition: a clinician diagnosis of asthma and $\geq 2$ respiratory tract infections/preceding year requiring oral antibiotics or on prophylactic antibiotics. Control definition: a clinician diagnosis of asthma, no evidence of recurrent infections and not prescribed prophylactic antibiotics. A 1:1 case-control ratio was used. 71 cases and 71 controls were identified. The antibiotic use, physiology, CT imaging, immunoglobulins and pneumococcal serotype meta-data were evaluated. Model based cluster analysis was performed to phenotype the cases with no a priori assumption made on the number of clusters. Age, sex, age of onset, sputum eosinophil count and
Juniper Asthma Control Score were used as the input variables (Am J Respir Crit Care Med.2008 Aug 1;178(3):218-24).

Results The cases were predominately female (69\%), obese with recurrent infections (mean:4.25/preceding year) and had an impaired asthma-related quality life compared to controls $(\mathrm{p}=$ 0.0285). Cluster analysis identified three groups. Cluster 1: male, eosinophilic, on oral corticosteroids with a low IgM, had poor lung function and bronchial wall thickening and bronchiectasis on CT. Cluster 2: female with a blood neutrophilia and preserved lung function. Cluster 3: female, non-atopic with impaired asthma control, a low IgG and air trapping on CT. $63.3 \%$ of patients on prophylactic antibiotics $(n=49)$ had a reduction in infective exacerbation frequency. The proportion of patients on antibiotics within each cluster and response was similar.

Conclusion Three subphenotypes of asthma with recurrent infections have been identified. Further immunpathological studies to evaluate the mechanism of infection in each subphenotype, the host microbiome and response to antimicrobials are required.

\section{P69 AN EXPLORATORY STUDY TO INVESTIGATE THE RELATIONSHIP BETWEEN FRACTION OF EXHALED NITRIC OXIDE (FENO) HOME MONITORING AND EOSINOPHILIC AIRWAY INFLAMMATION IN ADULTS WITH SEVERE ASTHMA}

CR Nanda, A Singapuri, M Soares, W Monteiro, S Siddiqui, S Gonem. Institute for Lung Health, Leicester, UK

\subsection{6/thoraxjnl-2015-207770.206}

Introduction $\mathrm{FeNO}$ is a non-invasive surrogate marker of corticosteroid-responsive airway inflammation that may be measured using small portable devices. We aimed to determine (i) the reliability and feasibility of twice-daily FeNO home monitoring in adults with severe asthma, as well as (ii) to explore the relationship between serial FeNO measurements and gold standard markers of eosinophilic airway inflammation.

Methods Ten patients with severe asthma (BTS treatment steps 4/5) were recruited from the Difficult Asthma Clinic at Glenfield Hospital. Patients were provided with portable FeNO monitors (NOBreath, Bedfont Scientific Ltd., Maidstone, UK) for a period of eight weeks, and asked to record twice-daily FeNO (at a flow rate of $50 \mathrm{ml} / \mathrm{s}$ ) and PEF readings, as well as daily visual analogue scores for cough, breathlessness and wheeze, using paper diaries. They attended fortnightly visits during the study period, at which they underwent sputum induction and full blood count. Results Nine patients completed the study. The median (range) intraclass correlation coefficient of triplicate FeNO measurements was $0.83(0.78-0.92)$ for morning measurements and $0.82(0.71-0.97)$ for evening measurements. There was a median of 7.1\% missing data (range 2.7 - 14.3\%). FeNO measurements correlated strongly with sputum and blood eosinophil counts, with the strongest correlations observed with a 9-day FeNO moving average, and a lag time of -1 day for sputum eosinophils $(\mathrm{r}=0.571, \mathrm{p}<0.001)$ and -2 days for blood eosinophil counts $(r=0.691, p<0.0001)$, suggesting that changes in sputum and blood eosinophil counts tended to precede changes in FeNO by 1 and 2 days respectively. In contrast there were no consistent relationships seen between FeNO and either PEF or visual analogue scores.

Conclusion Home monitoring of FeNO is feasible and the measurements are repeatable. Daily FeNO measurements correlate 
strongly with sputum and blood eosinophil counts, and are most useful when a moving average is taken over approximately 9 days. Further studies are required to determine if daily FeNO measurements may have a role in predicting loss of asthma control or exacerbations.

\section{\begin{tabular}{|l|l}
\hline P70 BLOOD EOSINOPHIL COUNTS IN NORMAL CONTROLS \\
\hline
\end{tabular} WITH NO HISTORY OF ALLERGIC DISEASE}

${ }^{1}$ A Anand, ${ }^{1} \mathrm{O}$ Ahmed, ${ }^{1} \mathrm{ID}$ Pavord, ${ }^{2} \mathrm{M}$ McClure, ${ }^{2} \mathrm{~B}$ Ferry, ${ }^{2}$ SR Pavord. ${ }^{1}$ University of Oxford, Oxford, UK; ${ }^{2}$ Oxford University Hospitals, Oxford, UK

\subsection{6/thoraxjnl-2015-207770.207}

Background The beneficial effect of corticosteroids and antiinterleukin (IL)-5 on exacerbations of airway disease becomes apparent at blood eosinophil counts above $0.15 \times 10^{9} / \mathrm{L}$, well within the normal range. One potential explanation is that the upper limit of the normal range is artificially high because studies have included patients with allergic disease and eosinophilic inflammation. We have assessed the normal range for blood eosinophil counts in volunteers with no self-reported history of allergic disease and compared this with the findings from a more traditional control population.

Methods We recruited 78 volunteers (15 male) with a mean age of 38.8 years. Volunteers with a self-reported history of asthma, allergic rhinitis and/or eczema were excluded. The differential cell count was carried out using sysmex $\mathrm{XN}$ analyser and serum IgE measured using automated enzyme immunoassay by Phadia Immunocap equipment. Result were compared with an unscreened population $(\mathrm{n}=120)$ used to calculate our local normal ranges.

Results One outlier value of $0.79 \times 10^{9} / \mathrm{L},(>5 \mathrm{SD}$ above the mean) was excluded from further analysis. In the remainder the mean blood eosinophil count was $0.15 \times 10^{9} / \mathrm{L}$ with an upper limit of normal range of $0.27 \times 10^{9} / \mathrm{L}$. Volunteers with no selfreported history of allergic disease but an $\operatorname{IgE}>120 \mathrm{iu} / \mathrm{L}$ and/or positive specific IgE to house dust mites or grass were not statistically different. The mean blood eosinophil count in the laboratory population was $0.19 \times 10^{9} / \mathrm{L}$ (p 0.018 vs our population) and the upper limit of normal range $0.42 \times 10^{9} / \mathrm{L}$.

Conclusions The upper limit of the normal range for blood eosinophil count is lower in a population who have no clinical history of allergic disease.

\section{P71 THE RELATIONSHIP BETWEEN THE LEICESTER COUGH QUESTIONNAIRE, EOSINOPHILIC AIRWAY INFLAMMATION AND ASTHMA PATIENT RELATED OUTCOME MEASURES IN SEVERE ASTHMA}

'S Siddiqui, 'R Free, ${ }^{1} \mathrm{P}$ Bradding, ${ }^{2} \mathrm{~L}$ McGarvey. 'Leicester NIHR Respiratory Biomedical Research Unit. University of Leicester, Department of Infection, Immunity and Inflammation, Leicester, UK; ${ }^{2}$ Queen University Belfast, School of Medicine, Dentistry and Biomedical Sciences., Belfast, Ireland

\subsection{6/thoraxjnl-2015-207770.208}

Background Severe asthma is characterised by a variety of symptoms which include chronic cough, although the mechanisms responsible are poorly understood. Modulation of cough reflex sensitivity by eosinophilic airway inflammation is considered likely but has not been well studied. Likewise the impact of chronic cough on patients health status is not well known as existing asthma patient related outcome instruments such as the
Juniper Asthma Control Score (ACQ-6), Asthma Quality of Life Questionnaire (AQLQ) were not primarily designed to capture cough and its related morbidity in asthma. We sought to evaluate (i) the Leicester Cough Questionnaire (LCQ) in a severe asthma population, (ii) the relationship between the Leicester Cough Questionnaire (LCQ) and the ACQ-6, AQLQ and (iii) airway inflammation in sputum in severe asthma patients.

Methods 312 patients [mean (SD) age of 60.4(16.2) years, median (IQR) GINA treatment score 5[4-5] and median (IQR) sputum eosinophil percentage of $3.0(0.5-16.6)]$ attending the Leicester difficult asthma service were evaluated with the LCQ, ACQ-6 and AQLQ at a single clinical visit. Induced sputum samples were also acquired at the same visit for differential cell count.

Results The LCQ demonstrated the following distribution properties: mean 15.30, standard deviation 4.49, range 4.04-21 and $10^{\text {th }}$ percentile point of 8.52 . Domain specific scores were LCQ (physical) 4.8(1.45), LCQ (psychological) 5.30 (1.55) and LCQ (social) 5.19(1.67). There were modest correlations between LCQ and ACQ-6 ( $r=-0.60 ; \mathrm{p}<0.001)$ but not with AQLQ ( $\mathrm{r}$ $=-0.067$ ). There was no correlation between LCQ and sputum eosinophilis/neutrophils.

Discussion Severe asthma is associated with a high degree of cough related morbidity that appears to be independent of eosinophilic airway inflammation and not captured fully by available patient reported outcome instruments. Further research is required to determine the validity of the LCQ and its responsiveness in severe asthma populations.

\section{\begin{tabular}{|l|l}
\hline P72 & T2 BIOMARKERS RELATE TO EXACERBATIONS AND
\end{tabular} CONTROL IN REFRACTORY ASTHMA}

S Srivastava, A Sahal, A Mansur. Birmingham Heartlands Hospital, Birmingham, UK

\subsection{6/thoraxjnl-2015-207770.209}

Background T2 biomarkers have been shown to predict responsiveness to corticosteroids and possibly relate to asthma exacerbations and control. T2 biomarkers in the form of fraction exhaled nitric oxide (FeNO), peripheral blood eosinophils (PBE), and serum periostin are easier to measure and probable surrogate markers for induced sputum eosinophilia (ISE). The relationships between $\mathrm{PBE}, \mathrm{FeNO}$ and periostin particularly in refractory asthma have been conflicting. A composite score of T2 biomarkers has also been postulated to predict exacerbations and may be more sensitive. ${ }^{1}$

Aim To explore the relationship between the T2 biomarkers individually, and in the form of composite score to asthma exacerbations and control.

Methods Unselected consecutive patients with confirmed diagnosis of refractory asthma (ATS) attending a tertiary severe asthma centre were recruited following an informed consent. Participants were evaluated for the followings: demographics, exacerbations requiring corticosteroids in the preceding 12 months, asthma control questionnaire (Juniper ACQ7), lung function, FeNO, PBE, and periostin measurement. The composite T2 score of all the 3 biomarkers was calculated as previously reported (reference). Statistical analyses were conducted using MedCalc software.

Results One-hundred and fifteen patients were recruited with mean age 45 yrs, 88 (69.8\%) females, mean inhaled corticosteroids $(\mathrm{BDP}$ equivalent $)=1,647 \mu \mathrm{g} / \mathrm{day}$, on maintenance $\mathrm{OCS}=$ 